\title{
Design, Optimization and Verification of 16S rRNA Oligonucleotide Probes of Fluorescence in-situ Hybridization for Targeting Clostridium spp. and Clostridium kluyveri
}

\author{
Lintao $\mathrm{Hu}^{1,2}$, Jun Huang ${ }^{1,2}$, Hui $\mathrm{Li}^{1,2}$, Yao $\mathrm{Jin}^{1,2}$, Chongde $\mathrm{Wu}^{1,2}$, and Rongqing Zhou ${ }^{1,2,3 *}$ \\ ${ }^{1}$ College of Light Industry, Textile and Food Engineering, Sichuan University, Chengdu 610065, P.R. China \\ ${ }^{2}$ Key Laboratory of Leather Chemistry and Engineering, Ministry of Education, Sichuan University, Chengdu 610065, P.R. China \\ ${ }^{3}$ National Engineering Research Center of Solid-State Manufacturing, Luzhou 646000, P.R. China
}

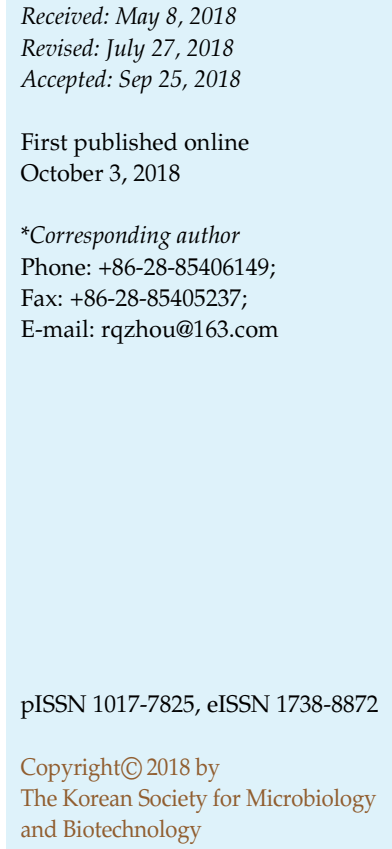

Fluorescence in-situ hybridization (FISH) is a common and popular method used to investigate microbial communities in natural and engineered environments. In this study, two specific 16S rRNA-targeted oligonucleotide probes, CLZ and KCLZ, were designed and verified to quantify the genus Clostridium and the species Clostridium kluyveri. The optimal concentration of hybridization buffer solution for both probes was 30\% (w/v). The specificity of the designed probes was high due to the use of pellets from pure reference strains. Feasibility was tested using samples of Chinese liquor from the famed Luzhou manufacturing cellar. The effectiveness of detecting target cells appears to vary widely in different environments. In pit mud, the detection effectiveness of the target cell by probes CLZ and KCLZ was $49.11 \%$ and $32.14 \%$, respectively. Quantitative analysis by FISH technique of microbes in pit mud and fermented grains showed consistency with the results detected by qPCR and PCR-DGGE techniques, which showed that the probes CLZ and KCLZ were suitable to analyze the biomass of Clostridium spp. and C. kluyveri during liquor fermentation. Therefore, this study provides a method for quantitative analysis of Clostridium spp. and C. kluyveri and monitoring their community dynamics in microecosystems.

Keywords: Fluorescence in-situ hybridization, 16S rRNA, specific oligonucleotide probe, Clostridium, Clostridium kluyveri

\section{Introduction}

Chinese liquor is one of the most famous distilled spirits in the world, thanks to its characteristic aroma endowed by unique brewing techniques [1,2]. Among the many different types of Chinese liquor, Luzhou liquor has an important feature in its crucial dependence on the quality of the pit mud (PM) used in its production. PM is a special kind of clay that provides a suitable habitat for the growth of brewing microbes. Similar to many other niches, such as soil and sludge, the composition of the microbial community in PM is very complex. Various methods, including both culture-dependent and independent measurements, have been used to explore its microbial diversity and their successions during the brewing process over the last three decades. It was reported previously and confirmed by independent methods that this microbial diversity was closely connected with pit ages [3,4]. In the last three decades, various molecular biology technologies (PCRbased, fluorescence in-situ hybridization (FISH), etc.) have also been used to investigate the characteristics of the microbial communities in PM [5, 6]. The results from these studies suggest that Clostridium plays an important role in the process of brewing. Hexanoic acid, one of the major metabolites of the brewing process, is the precursor of ethyl hexanoate. Ethyl hexanoate has a fruity, floral, sweet aroma and is one of the main volatile compounds in liquor produced in Luzhou, China, as well as other types of 
Chinese liquor [7-11]. Therefore, the abundance of Clostridium, especially Clostridium kluyveri, in PM and fermented grains, was deemed as one of the important evaluation criteria for bioactivity $[5,6,8]$. Clostridium, which includes more than 100 species, are classified as anaerobic bacteria with the majority remaining uncultured so far [9, 10], apart from a few of species, such as Clostridium spp. and C. kluyveri $[12,13]$. Therefore, close attention has been paid to investigate the characteristics of Clostridium during the process of brewing [11, 14, 15]. However, it was not yet conducive to estimate the evolution of the microbial community in this process quantitatively and directly, or to in-situ screen functional Clostridium in PM and analyze the impact of the brewing process on the microbial community in PM. The qPCR method was used to determine copies of the 16S rRNA gene of C. kluyveri in PM quantitatively [12]. However, it was still difficult to accurately characterize the composition of Clostridium in complex systems due to the influence of various factors such as DNA extraction, amplification efficiency, and inability to distinguish activity. FISH with rRNA-targeted oligonucleotide probes facilitates the rapid and specific identification of individual microbial cells in their natural environments [16]. The advantage of FISH is that it allows us to visualize, identify, enumerate and locate the cells of specific microbe groups in both cultured and uncultured states [17]. It has been used to characterize archaeal communities in complex environments $[18,19]$. In our previous research we used FISH and various order-specific oligonucleotide probes to characterize the microbial communities in PM [20-24]. Moreover, we were also able to quantitatively estimate the composition of four orders (methanosarcinales, methanobacteriales, methanomicrobiales and methanococcales) in the methanogenic archaeal communities of various aged PM [25]. These results clearly showed that FISH can be used to evaluate community diversity and dynamics during Chinese liquor production, in which choosing the appropriate oligonucleotide probes is a critical step.

The aim of this study was to develop two specific oligonucleotide probes (CLZ and KCLZ) targeting Clostridium spp. and C. kluyveri, respectively. Moreover, we optimized the concentration of hybridization and also examined the feasibility and sensitivity of genus and species. We next carried out validation of the specificity and accuracy of the probes. Using PM spiked with reference strain, we were also able to prove real-time qPCR and PCR-DGGE techniques as well as establishing the effectiveness of these probes.

\section{Materials and Methods}

\section{Microbial Strains and Culture Conditions}

Microbial strains used in the present research were purchased from the China Center of Industrial Culture Collection (CICC), except for Lactobacillus casei (ATCC 393) (purchased from the American Type Culture Collection). These strains included three strains of Clostridium and two strains of non-Clostridium. C. kluyveri (CICC 8022) was cultivated in ethanol/acetate/succinate/yeast extract (EASY) medium according to a culture method described by Weiner et al. [26]. Clostridium butyricum (CICC 10350) was cultivated in medium composed of $5.0 \mathrm{~g}$ corn steep flour, $20 \mathrm{~g}$ glucose, $1 \mathrm{~g}\left(\mathrm{NH}_{4}\right)_{2} \mathrm{SO}_{4}, 1.24 \mathrm{~g} \mathrm{NaHCO}_{3}, 0.2 \mathrm{~g} \mathrm{MnSO}_{4} \cdot \mathrm{H}_{2} \mathrm{O}, 0.2 \mathrm{~g}$ $\mathrm{MgSO}_{4} \cdot \mathrm{H}_{2} \mathrm{O}, 0.2 \mathrm{~g} \mathrm{MgSO}_{4} \cdot 7 \mathrm{H}_{2} \mathrm{O}, 0.02 \mathrm{~g} \mathrm{CaCl}_{2}$ per liter at $35^{\circ} \mathrm{C}$ in an anoxic environ. TGY broth ( $3 \%$ trypticase, $2 \%$ glucose, $1 \%$ yeast extract, and $0.1 \%$ L-cysteine) was used for Clostridium perfringens (CICC 22949) vegetative growth (initial pH 7.2-7.4). MRS (QDRS BIOTEC, China) broth or agar medium was used for L.casei (ATCC 393) vegetative growth (initial pH 6.2-6.4). Bacillus subtilis (CICC 20633) was cultivated in tryptic soy broth or agar (Beijing Borunlaite Science \& Technology, Co., Ltd, China).

\section{Sampling and Pretreatment}

These samples were obtained from the cellar located at Luzhou Laojiao Co., Ltd. (Luzhou City, Sichuan Province, China), which has been brewing liquor for more than 300 years. Sampling was performed according to the stratified random method described by Ding et al. [27]. The samples obtained from the pit mud, fermented grains and mixture (composed of Daqu powder, fermented grains, rice hull and broken sorghum) were abbreviated as PM, BFG, and SFG, respectively. SFG was steamed for distilling liquor and liquefying and saccharifying starch. These samples were packaged in a sterile polyethylene bag and immediately stored at $-20^{\circ} \mathrm{C}$ until analysis.

The samples were also pretreated prior to examination according to the method described by Li et al. [22].

\section{Probe Design}

Specific oligonucleotide probes for genus Clostridium and species C. kluyveri were designed according to the method previously described $[28,29]$. Sequence manipulations were carried out by

Table 1. Oligonucleotide sequences and targeted microbes of the probes designed in this study.

\begin{tabular}{lll}
\hline Probe & \multicolumn{1}{c}{ Target group } & \multicolumn{1}{c}{ Sequence $\left(5^{\prime}-3^{\prime}\right)$} \\
\hline KCLZ & Clostridium kluyveri & $5^{\prime}$-CY3-CCTGCACACCCTTTACGCCCAGTAATTCCGGACAA - $3^{\prime}$ \\
CLZ & Clostridium & $5^{\prime}$-CY3-GGCTACCTTGTTACGACTTCACCCCA- $3^{\prime}$ \\
\hline
\end{tabular}


Primer Premier 6.0 (Premier Biosoft International, Canada, 2002. 3). The sequences were initially aligned with $16 \mathrm{~s}$ rRNA gene sequences available in the GenBank database (http://www.ncbi.nlm.nih.gov) by MEGA 6.0 (www.megasoftware.net) and adjusted manually. Specific oligonucleotide probes for genus Clostridium and species C. kluyveri shown in Table 1, were authorized to Sangon Biotech (China) for synthesis.

\section{Fluorescence in-situ Hybridization}

FISH experiments with the CLZ and KCLZ probes were performed as described previously [24,30]. The pretreated pit mud was fixed in a $4 \%$ freshly-prepared paraformaldehyde at $4^{\circ} \mathrm{C}$ for $16 \mathrm{~h}$ and then suspended in PBS buffer. After intensive mixing, the pellets were collected by centrifugation $\left(10,000 \mathrm{~g}, 4^{\circ} \mathrm{C}, 10 \mathrm{~min}\right.$, Anke, GL-20G- II), and resuspended in $10 \mathrm{ml}$ of sodium pyrophosphate solution $(3.8 \mathrm{mM})$. The suspension was diluted to $10^{7}-10^{8} \mathrm{CFU} / \mathrm{ml}$, and $20 \mu \mathrm{l}$ of this dispersed sample was doped onto a poly-L-Lysine-coated slide. The slide was air-dried at $37^{\circ} \mathrm{C}$ for $2 \mathrm{~h}$, and then dehydrated by successive passages through $50 \%$, $80 \%$, and $100 \%$ ethanol, respectively (each for $3 \mathrm{~min}$ ). The slides were incubated at $37^{\circ} \mathrm{C}$ for $30 \mathrm{~min}$ in $50 \mu$ lysozyme solution $(10 \mathrm{mg} / \mathrm{ml})$, washed with sterile distilled water, and then dehydrated and air-dried by the process described above. Hybridization was performed at $42^{\circ} \mathrm{C}$ for $2 \mathrm{~h}$ with hybridization buffer (0.9 M NaCl, 20 mM Tris-HCl (pH 7.4), 0.01\% (w/v) SDS) containing the designed probes (KCLZ, CLZ), in which different concentrations of formamide were added. For the EUB338 (5'GCTGCCTCCCGTAGGAGT-3') probe, the optimized concentration $(20 \%(\mathrm{w} / \mathrm{v}))$ was employed as related research has already been reported previously [31]. Slides were washed twice in $50 \mathrm{ml}$ of prewarmed washing buffer $(20 \mathrm{mM}$ Tris- $\mathrm{HCl}(\mathrm{pH} 7.2), 10 \mathrm{mM}$ EDTA, $0.01 \%(\mathrm{w} / \mathrm{v}) \mathrm{SDS}, 0.3 \mathrm{M} \mathrm{NaCl})$ at $48^{\circ} \mathrm{C}$ for $30 \mathrm{~min}$, and subsequently washed with distilled water and air-dried in the dark after hybridization. Finally, the slides were mounted in an anti-fade solution, and observed using a fluorescence microscope (BX-51, Nikon, Japan) with a WG filter for Cy3 (excitation 534$558 \mathrm{~nm})$.

After optimizing the formamide concentration, a FISH experiment was performed using firstly the pure culture of reference strains listed above and the mixture of these pure cultures, respectively. The pure culture of C. kluyveri (CICC 8022) was then added into a PM sample. Meanwhile, the mixtures of pure culture were added into other PM samples with different volumes $(0.2,0.4,0.6,0.8$, and $1 \mathrm{ml}$ ). After sample pretreatment, the samples were added onto the slides and put into the microscope for FISH counting analysis. Fifteen fields for each sample were randomly counted using FISH 3.0 software (Imstar, France). The fluoro-stained cell concentration was calculated as follows:

$$
\mathrm{N}=n \times \frac{s_{1}}{S_{2}} \times \frac{v_{1}}{v_{2}} \times D
$$

Where $\mathrm{N}$ is the cell number in $1 \mathrm{~g}$ of dried pit mud (cells/g), $\mathrm{n}$ is the mean number observed in a field, $s_{1}$ is the spreading area $\left(\mu \mathrm{m}^{2}\right), s_{2}$ is the field area $\left(\mu \mathrm{m}^{2}\right), v_{1}$ is the spreading volume $(\mu \mathrm{l}), v_{2}$ is the total volume $(\mu \mathrm{l})$ and $D$ is the dilution rate.

The samples originated from pit, including PM, BFG, and SFG, were pretreated, and FISH experiments were carried out according to the process described above using hybridization buffer containing various probes from previous articles [22]. The fluoro-stained cell concentration was also calculated.

\section{Quantitative Characterization of Total Bacteria and C. kluyveri Based on qPCR Analysis}

Whole genomic DNA was extracted according to the methods of Zhou et al. [32]. SybrGreen qPCR Master Mix (Toyobo Life Science, China) was used for all measurements. The qPCR reaction volume was fixed at $20 \mu \mathrm{l}$, containing $10 \mu \mathrm{M}$ of each primer, $2 \times$ reaction buffer and $2 \mu \mathrm{l}$ of DNA. The primers were 357F (5'CTACGGGAGGCAGCAG-3')/517R (5'-ATTACCGCGGCTGCT GG-3') and CloKly1F (5'-GAGGGAGCAAATCT AAAAACTGC3')/CloKly1R (5'-CCTCCTTGGTTAGACTACGGACTT-3') for the amplification of eubacteria and C. kluyveri, respectively. Amplification occurred in a StepOnePlus Real-Time PCR System (Thermo Scientific, USA) using the following program: $3 \mathrm{~min}$ at $95^{\circ} \mathrm{C}, 45$ cycles of $15 \mathrm{sec}$ at $95^{\circ} \mathrm{C}, 20 \mathrm{sec}$ at $57^{\circ} \mathrm{C}$, and $30 \mathrm{sec}$ at $72^{\circ} \mathrm{C}$.

The PCR product of primers specifically for eubacteria and C. kluyveri was then amplified by conventional PCR using primer 338F (5'-ACTCCTACGGGAGGCAGCAG-3')/518R (5'-ATTACC GCGGCTGCTGG-3') and Clokly1F/CloKly1R, respectively, according to the methods described by Lee et al. [33], and were denoted as pGEM-C and pGEM-D. A 10-fold serial dilution series of the pGEM-C and pGEM-D, ranging from $1 \times 10^{5}$ to $1 \times 10^{9}$ copies $/ \mu \mathrm{l}$, was used to construct the calibration curve for C. kluyveri and total eubacteria, respectively. The concentration of the plasmid was measured using a fluorometer and the corresponding copy number was calculated using the following equation [34]:

$$
\text { DNA }(\text { copy })=\frac{6.02 \times 10^{23}(\mathrm{copy} / \mathrm{mol}) \times D N A \text { amount }(g)}{D N A \text { length }(\mathrm{bp}) \times 660(\mathrm{~g} / \mathrm{mol} / \mathrm{bp})}
$$

Where Avogadro's number is $6.02 \times 10^{23}$ copies $/ \mathrm{mol}$, and the average molecular weight of one DNA base pair (bp) is $660 \mathrm{~g} / \mathrm{mol}$. The $C_{T}$ values obtained from analyzing serially diluted standard solution are plotted versus the logarithm of the corresponding initial concentrations. The $\mathrm{C}_{\mathrm{T}}$ values were plotted against the logarithm of their initial template copy numbers. A linear regression of the plot forms the calibration curve so that the concentration of target sequence in an unknown sample can be estimated by interpolation.

\section{Characterization of the Microbial Community Based on PCR- DGGE Analysis}

Whole genomic DNA was extracted by the same method as mentioned before. A nested PCR was employed, and all PCR primers used in this study derived from a previous study [23]. Before DGGE analysis, PCR products were examined by electrophoresis on $1 \%$ agarose gels. The DCode Universal Mutation 
Detection System (Bio-Rad, USA) was used for sequence-specific separation of PCR amplified fragments, and DGGE was performed according to the previously reported protocols [30]. Representative bands observed on the DGGE profiles were excised, eluted at $4^{\circ} \mathrm{C}$ in ultrapure water, and then re-amplified. PCR products were purified with a universal PCR purification kit (Tiangen, China) and sent to a commercial sequencing company for cloning and sequencing (Sangon, China). The sequences were then compared with 16S rRNA gene sequences available in the GenBank by BLAST (http://www.ncbi.nlm.nih.gov/BLAST) and RDP CLASSIFIER (http://rdp.cme.msu.edu/index.jsp) searches to identify their closest phylogenetic relatives.

The unique sequences obtained from this study were deposited in GenBank with the following accession numbers: MG729405MG729417 and MG729419-MG729437 (the 16S rRNA V3 region sequences of eubacteria).

\section{Data Analysis}

All assays were conducted in triplicate, and the results were expressed as mean \pm standard deviations. Analysis of variance (ANOVA) was used to test the significance of each assay. Results were considered to be statistically significant at $p<0.05$. Community diversity was determined using Quantity One Software, which was used to convert individual DGGE lanes to densitometric profiles. Simpson dominance index (D), Species richness (S), Evenness index (J) and the Shannon-Wiener index $(\mathrm{H})$ were determined based on the number and relative intensities (RIs) of bands, and were calculated using ZZSTAT V2010.

\section{Results and Discussion}

To design a specific oligonucleotide probe for the phylogenetic Clostridium group, BLAST and RDP CLASSIFIER were used to align and identify the obtained 16S rRNA sequences. A target site was identified in almost all available sequences of the Clostridium group, including Clostridium genus and C. kluyveri species from subgroup. An evolutionary distance tree of the Clostridium (Appendix) outlines the different Clostridium groups and the targets of probes used in the study. The KCLZ target site, with an adenosinethymine wobble at position, and the CLZ target site, with an adenosine-guanine wobble at position were listed in Appendix. The specificity and coverage of the new probes were evaluated and tested by SILVA [35]. The results revealed high specificity for both probes; the probe KCLZ exhibited a coverage of $4.3 \%$ and a specificity of $100 \%$ for the species "C. kluyveri," while the probe CLZ demonstrated a coverage of $31.7 \%$ and a specificity of $100 \%$ for the genus "Clostridium."

\section{Exploring the Feasibility of Quantitative Determination of Genus and Species Based on CLZ and KCLZ Probe}

Three environmental and six methodological factors should be taken into account for the detection of bacteria by the FISH technique [36]. In our present experiment, the three environmental factors (type of ecosystem, bacteria growth rate and dominant phylogenetic group) were of lesser importance. Among the methodological factors (fixative, fluorochrome, hybridization temperature, formamide concentration in hybridization buffer, $\mathrm{NaCl}$ concentration in wash solution and counting method), formamide concentration in hybridization buffer and $\mathrm{NaCl}$ concentration in wash solution are crucial to hybridization efficiency [37]. The hybridization of pretreated pit mud samples with both probes were performed using the hybridization buffer containing $10 \%, 20 \%, 30 \%, 40 \%, 50 \%, 60 \%$, and $70 \%$ formamide, respectively. The results showed that $30 \%$ formamide was the most efficient, which was similar to the results with EUB338 [24]. As shown in Fig. 1A, compared with $30 \%$ formamide contained in the hybridization buffer,

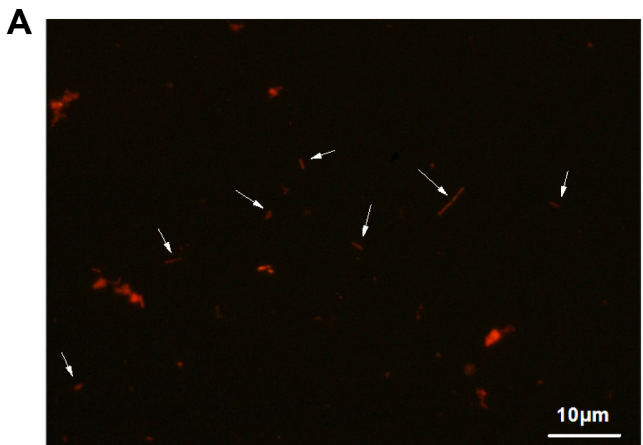

KCLZ

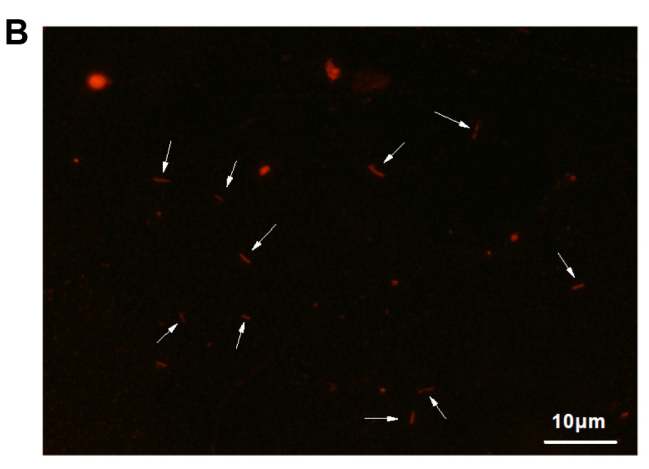

CLZ

Fig. 1. Epifluorescence micrographs of samples hybridized with KCLZ (A) and CLZ (B) probes in the hybridization buffer of optimal (30\%) concentration. 
the quantity of detected target microorganism (C. kluyveri) was lower, and the intensity of fluorescence background was weaker with $10 \%$ and $20 \%$ formamide content in the buffer. Formamide decreased the melting temperature by weakening the hydrogen bonds, thus enabling a lower temperature to be used with high stringency. Therefore, enhancing the formamide concentration increases the specificity of the hybridization advisably, but once it exceeds its optimal concentration it causes a drastic drop of the bound probe and signal intensity [38]. We observed a similar tendency in our result as the signal intensity dropped when $50 \%$ or $60 \%$ formamide was contained in the buffer. Meanwhile, the effect of formamide concentration on the hybridization efficiency of probe CLZ was similar to that of probe KCLZ. As shown in Fig. 1B, the quantity of detected target microorganism (Clostridium) was lower with $10 \%, 20 \%$, and $40 \%$ formamide concentration, and the intensity of fluorescence background was also weaker in these cases. Both the hybridization signals of the target and the intensity of fluorescence background were enhanced with $50 \%, 60 \%$, or $70 \%$ concentrations. When the hybridization buffer contained 30\% formamide, the fluorescence signals of the target were strong and evenly distributed, and the fluorescence background was relatively moderate, which allowed us to accurately quantify the number of Clostridium in complex systems. Otherwise, the quantity of detected target microorganisms using the CLZ probe was higher than with the KCLZ probe.

\section{Validating Specificity and Accuracy}

FISH experiments were performed using the CLZ and KCLZ probes, respectively, for the pretreated pure reference strain pellets. The results confirmed the genus-specificity of the CLZ and species-specificity of the KCLZ probes. The CLZ probe only hybridized with Clostridium, for example C. kluyeri, C. butyricum and C. perfringens, but could not hybridize with L. casei and B. subtilis, as shown in Fig. 2.

The detected result of the spiked pit mud with the pure culture of C. kluyeri $\left(1.12 \times 10^{9}\right.$ cells $\left./ \mathrm{ml}\right)$ was shown in Table 2. It indicates that the recovery rates of various probes were different: $83.05 \%, 49.11 \%$, and $32.14 \%$ for EUB338, CLZ and KCLZ, respectively. The efficiency of the KCLZ probe was slightly lower compared to that of the EUB338 and CLZ probes, which may have been caused by the behavior of different probes such as the position of the probe's target. As we know, EUB338, an eubacterial probe used in exploring the microbial abundance in complex systems, was also an overall median of $56 \%$ in enriched culture, ranged from $1 \%$ to $100 \%$ by the influence of

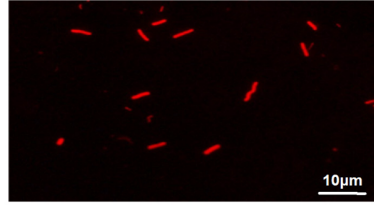

CLZ-Clostridium kluyveri

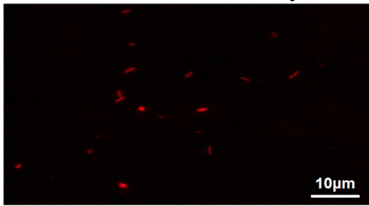

CLZ-Clostiridium butyricum
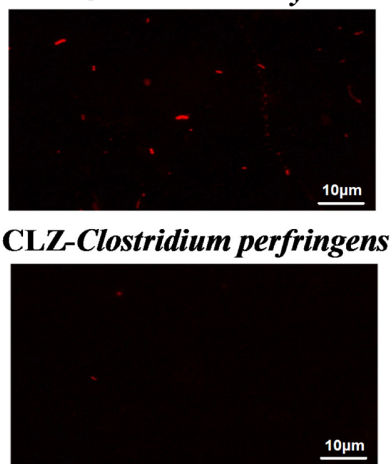

CLZ-Bacillus subtilis

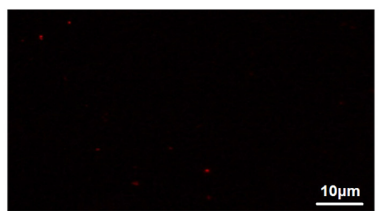

CLZ-Lactobacillus casei

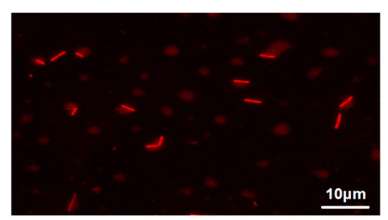

KCLZ-Clostridium kluyveri

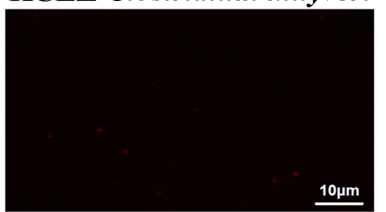

KCLZ-Clostiridium butyricum

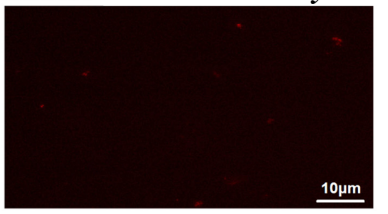

KCLZ-Clostridium perfringens
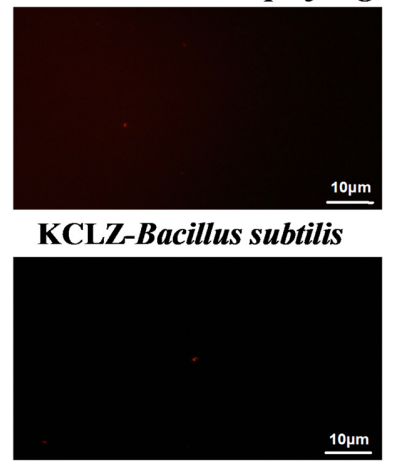

KCLZ-Lactobacillus casei
Fig. 2. Epifluorescence micrographs of the pure reference strains hybridized with CLZ and KCLZ probes.

various factors [36]. Furthermore, an increased trend in the spiked samples was revealed, although the difference between the spiked quantity and the detected quantity was not significant.

The sensitivity of the probes was verified by spiking pure cultures into pit mud. As shown in Fig. 3, the quantity of Clostridium in the spiked samples increased with the

Table 2. Concentration of microbes in blank samples and spiked samples.

\begin{tabular}{lcc}
\hline Probe & $\begin{array}{c}\text { Blank samples } \\
\left(\times 10^{9} \text { cells } / \mathrm{g}\right)\end{array}$ & $\begin{array}{c}\text { Spiked samples } \\
\left(\times 10^{9} \text { cells } / \mathrm{g}\right)\end{array}$ \\
\hline CLZ & $1.06 \pm 0.09$ & $1.61 \pm 0.12$ \\
KCLZ & $0.87 \pm 0.10$ & $1.23 \pm 0.09$ \\
EUB338 & $1.81 \pm 0.41$ & $2.74 \pm 0.33$ \\
\hline
\end{tabular}



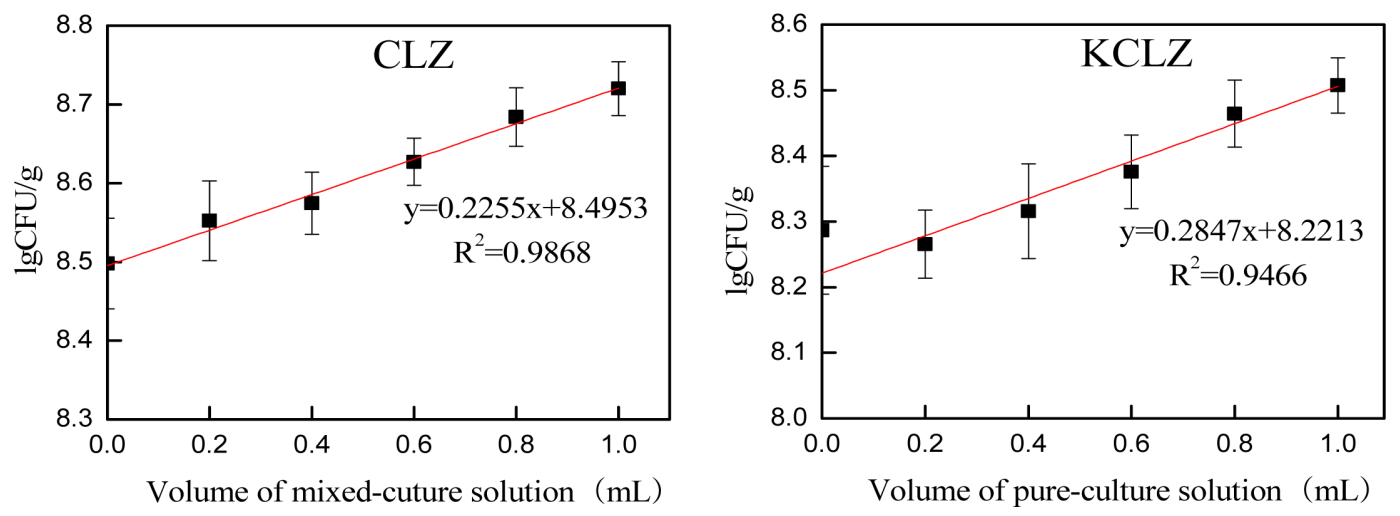

Fig. 3. The effects of different volumes of the mixtures of pure culture on the Clostridium spp. and C. kluyeri in the pit mud.

volume of the culture that formerly was a mixture, which included three strains of Clostridium (the proportion of C. kluyeri, C. butyricum and C. perfringens was $32.21 \%, 29.98 \%$ and $37.81 \%$ in the mixture of pure culture, respectively) and later was pure C. kluyeri.

These probes were used to quantify Clostridium spp. and C. kluyeri in pit mud samples taken from three different pits, which all had the same age. They were also used for the samples of BFG and SFG from the same pit. As shown in Table 3, no significant difference between the samples in the same niches (pit mud) was found for the results detected by various types of used probes, including the EUB338, CLZ, and KCLZ probes, but discrepancies between the samples originated from different phases in the same pit were observed. BFG was regarded as a microaerobic phase structured with a large number of voids, and the properties differed from those of PM. Compared with PM, it may not be suitable for propagating Clostridium spp. or C. kluyeri. Therefore, the concentrations of both in BFG were lower than those in pit mud, similar to the result obtained by Ding, et al. [27]. Nevertheless, the proportion of Clostridium spp. and C. kluyeri in SFG decreased from $36.9 \%$ to $27.1 \%$, and $17.9 \%$ to $12.3 \%$, respectively, although the quantity of eubacterium and the microbes detected by the CLZ and KCLZ probes were slightly lower than that in BFG. It may be caused by various factors, such as added
Daqu powder, loss of moisture, etc. It was noteworthy that C. kluyeri was detected by the KCLZ probe even if the fermented grains were placed in an aerobic environment for more than four hours, and then steamed at atmospheric pressure for more than $60 \mathrm{~min}$. A similar result, the thermal death of a pure culture of E. coli by autoclave was also observed in our preliminary test. But the fluorescence intensity was weak and the number of cells was low through the FISH technique. The possible reason was that E. coli was more resistant to heat than other bacilli, it just led to lower cell activity and no death. It is one of the major features that FISH exhibits to detect physiologically active bacteria in relatively nutrient-rich environments [39]. One can speculate that, due to the thermal denaturation of RNA, the hybridization activity with probes decreased, but no there was target loss for FISH analysis. Therefore, this nonholonomic RNA residual in the samples may explain the overestimation of specific-target genus and species. However, the result shows that the efficiency of designed species-specific probes was suitable to characterize quantitatively the abundance of C. kluyeri in the sample.

\section{Validating Based on Quantitative Analysis of qPCR}

The 16S rRNA gene copies of C. kluyeri and eubacteria in pit mud and fermented grains samples were quantitatively analyzed by using specific primers CloKly1F/CloKly1R

Table 3. Concentration of microbes in the different pit mud samples from pits of the same age, BFG samples and SFG samples.

\begin{tabular}{cccccc}
\hline Probe types & $1^{\mathrm{a}}\left(\times 10^{7}\right.$ cells $\left./ \mathrm{g}\right)$ & $2^{\mathrm{b}}\left(\times 10^{7}\right.$ cells $\left./ \mathrm{g}\right)$ & $\mathrm{A}^{\mathrm{c}}\left(\times 10^{7}\right.$ cells $\left./ \mathrm{g}\right)$ & $\mathrm{BFG}\left(\times 10^{7}\right.$ cells $\left./ \mathrm{g}\right)$ & $\mathrm{SFG}\left(\times 10^{7}\right.$ cells $\left./ \mathrm{g}\right)$ \\
\hline EUB338 & 264 & 281 & 205 & 0.84 & 1.55 \\
CLZ & 102 & 98 & 73 & 0.31 & 0.42 \\
KCLZ & 65 & 67 & 44 & 0.15 & 0.19 \\
\hline
\end{tabular}

\footnotetext{
${ }^{\mathrm{a}, \mathrm{b}}$ and ${ }^{\mathrm{c}}$ are pit mud samples taken from different pits of the same age, respectively.
} 
A
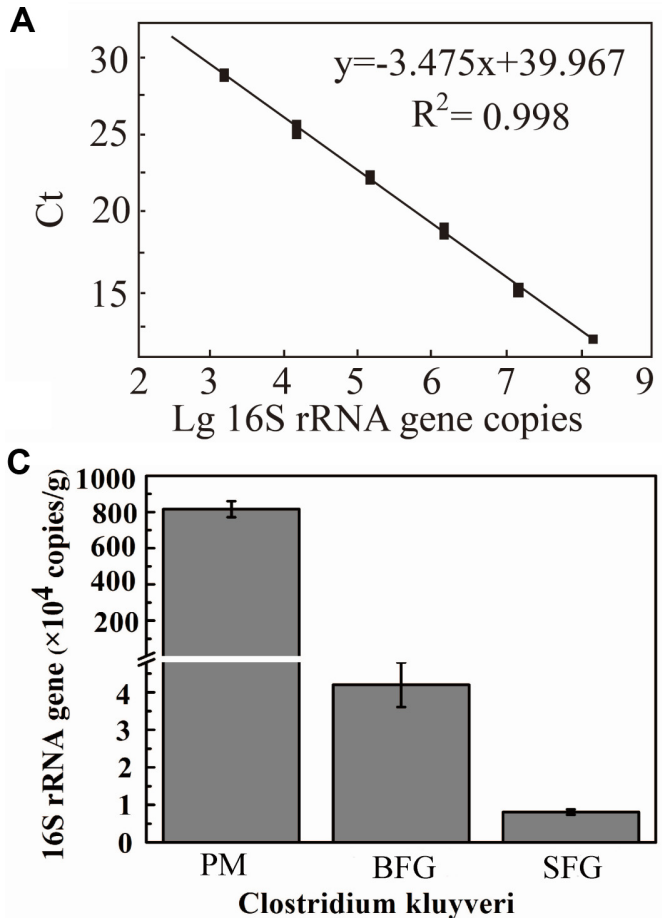

B

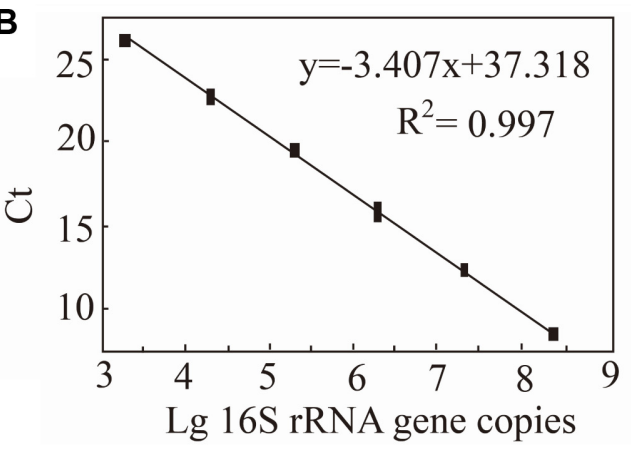

D

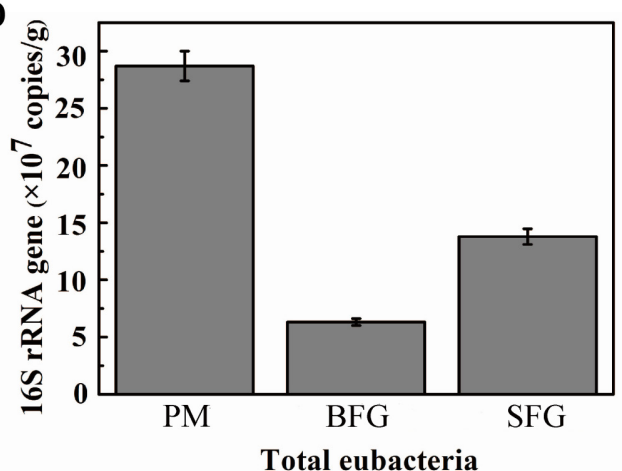

Fig. 4. Calibration curve of the real-time PCR for quantifying the $16 \mathrm{~S}$ rRNA gene copies of C. kluyveri (A) and eubacteria (B); $16 \mathrm{~S}$ rRNA gene copies of C. kluyveri (C) and eubacteria (D) in the samples obtained by qPCR.

and universal primer 357F/517R based on the 16S rRNA gene fragment of $C$. kluyeri. As shown in Fig. 4, the correlation coefficient $\left(R^{2}\right)$ values of the linear regression analysis based on the result of the calibration curves constructed for eubacteria and C. kluyeri were 0.998 and 0.997 , and the amplification efficiency (E) values were 0.966 and 0.940 according to the formula described by Knutsson, et al. [40]. The values of eubacteria quantity were $2.87 \times 10^{8}$ copies/g, wet, $6.31 \times 10^{7}$ copies/g, wet, and $1.37 \times 10^{8}$ copies $/ g$, wet, while those of $C$. kluyeri quantity were $8.16 \times 10^{6}$ copies $/ g$, wet, $7.88 \times 10^{4}$ copies $/ \mathrm{g}$, wet, and $1.52 \times 10^{4}$ copies $/ \mathrm{g}$, wet in PM, BFG and SFG samples, respectively. Their magnitude order was consistent with the results detected by the FISH technique, but $C$. kluyeri was abundantly present at levels of $2.843 \%, 0.125 \%$, and $0.011 \%$. On the other hand, those identified by FISH were $21.463 \%, 17.857 \%$, and $12.258 \%$, in PM, BFG and SFG, respectively. On the whole, the difference in C. kluyeri abundance among these microsystems was confirmed by the result of qPCR. Relative to the conventional PCR, qPCR is a more sensitive and precise method [41], and has been widely applied in many fields, such as ecology, diagnostics, etc. However, its precision has been also limited due to some inherent limitations and biases of PCR. Of these influencing factors [42], one of the major reasons why specific-groups were quantitively overestimated was nonviable amplificated DNA. In the process of brewing Chinese liquor in Luzhou, overestimation is a particular problem since pit mud is used, and the fermented grains are partly recycled into the pit. Therefore we can assert that it could be impossible to quantify target-genus and species in such complicated systems even if the estimation error derived from nonviable DNA could be mitigated by various developed approaches $[43,44]$.

\section{Validating Based on PCR-DGGE Analysis}

In order to assess qualitatively the accuracy of the designed probes, analysis based on PCR-DGGE for PM, BFG and SFG was performed. DGGE profiles of the eubacteria

Table 4. Diversity indices of eubacteria in the samples calculated from the DGGE banding patterns shown in Fig. 5A.

\begin{tabular}{llccc}
\hline \multirow{2}{*}{ Type } & \multicolumn{3}{c}{ Samples } \\
\cline { 3 - 5 } & & PM & BFG & SFG \\
\hline Eubacteria & Dominance index $(D)$ & 0.053 & 0.043 & 0.050 \\
& Species richness $(S)$ & 21 & 26 & 21 \\
& Evenness index $(J)$ & 0.983 & 0.984 & 0.991 \\
& Shannon-winner index $(H)$ & 2.993 & 3.204 & 3.017 \\
\hline
\end{tabular}



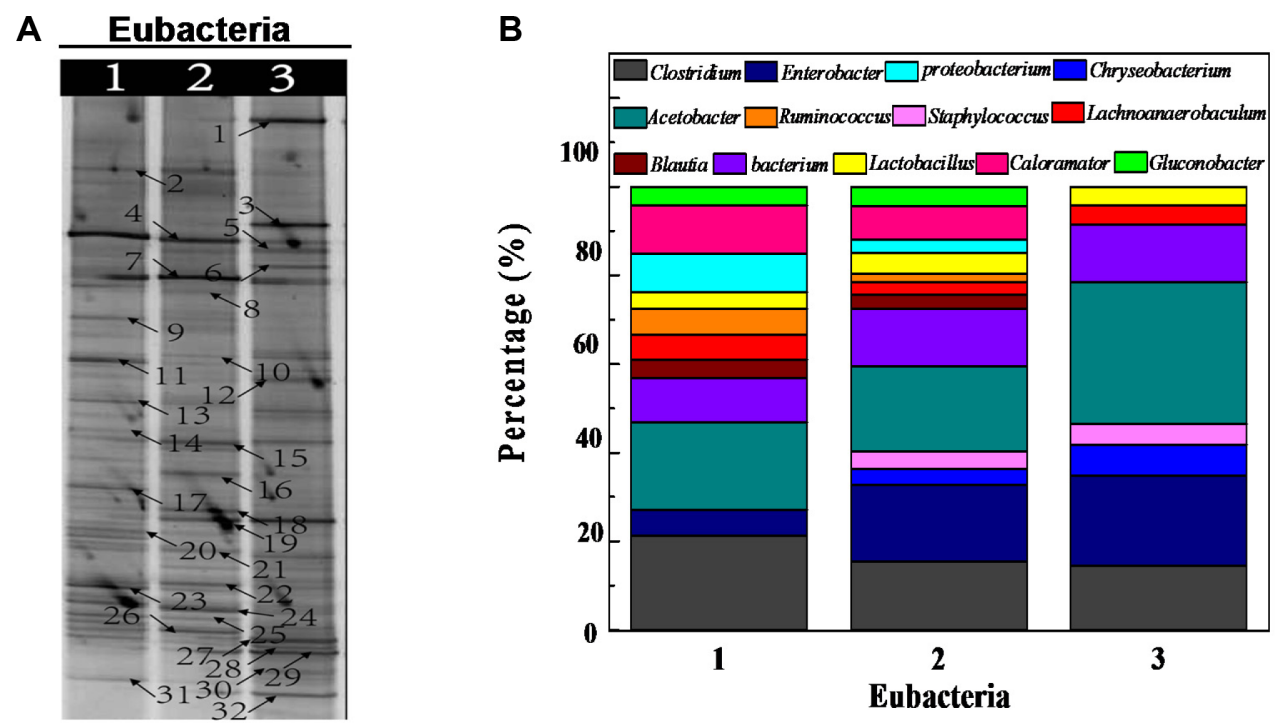

Fig. 5. DGGE profiles of the eubacteria microbes (A) from V3 region of $16 \mathrm{~S}$ rRNA obtained from 3 samples and the corresponding relative intensities (\%) of the eubacteria microbes $(\mathbf{B})$ in different samples.

The sample numbers 1-3 stand for PM, BFG and SFG, respectively.

are shown in Fig. 5A. Based on the analysis of DGGE profiles, diversity indices were determined. The results show that the difference of the microbial diversity index between PM, BFG and SFG was significant (As shown in Table 4). The Dominance index (D) of PM eubacteria was higher than BFG and SFG. D value and Evenness index (J) of BFG were lower than for SFG; as for the other indicators such as species richness $(\mathrm{S})$ and Shannon-winner index $(\mathrm{H})$, an opposite tendency can be observed between these two samples (BFG and SFG). For investigating the eubacterial community structure in PM, BFG, and SFG, representative bands from the PCR-DGGE were carefully excised, recovered and sequenced, and the results were shown in Table 5. The eubacterial DGGE pattern of the $16 \mathrm{~S}$ rDNA V3 regional gene fragment amplicons showed that different numbers of bands were detected in different samples. The similarity of all band sequences was nearly $>98 \%$ comparing with those available in the GenBank database. All eubacterial 16S rRNA sequences fell into three phyla (Proteobacteria, Bacterodetes and Fimincutes), comprising thirteen genera that were affiliated with six orders in five classes. Class $\alpha$-proteobacteria (order Rhodospirillales, bands $6,7,8,20,21,27,28,29,31$, and 32), class $\gamma$-proteobacteria (order Enterobacteriales bands 1, 2, 19, and 22) and proteobacterium (band 23) belonged to Proteobacteria. Next, Firmicutes included class Bacilli and class Clostridia, which involved three orders: Clostridiales, Bacillales and
Lactobacillales. Of these, Clostridiales (bands 4, 9, 13, 14, 17, 18, 30, and 31), Bacillales (band 5) and Lactobacillales (band 15) were affiliated with Firmicutes. Additionally, Bacterodetes had class Flavobacteriia, and Flavobacteriia included order Flavobacteriales (band 3). Of these bacteria, Acetobacter spp., Clostridium spp. and Enterobacter spp. were dominant. As shown in Fig. 5B, all eubacteria were detected in PM, and the abundance of Clostridium was $22 \%$, calculated based on the rate of their relative intensity detected (IR value). Clostridium genera was also detected in fermented grain samples, and the levels were $16 \%$ and $14 \%$ in BFG and SFG samples, respectively. The difference in Clostridium spp.'s abundance between these samples was also similar with FISH's. Species C. kluyveri was also identified in PM, BFG and SFG. In fact, C. kluyveri was often used to convert alcohol into a mixture of acetic, butyric and caproic acids with methane bacteria [45], as one of the important contributors forming the representative aroma and flavor compounds in Luzhou- type liquors.

In conclusion, the specific Clostridium spp. and C. kluyveri probe set CLZ and KCLZ, based on the available 16S rRNA genes, were designed and verified by experiment. The specificity of these designed probes was tested using pure reference strain pellets. Combined with the FISH technique, the Clostridium diversity of PM and fermented grain samples was successfully investigated, and showed the same regularity. The results were confirmed by qPCR and PCR- 
Table 5. Eubacteria sequence alignment with BLAST and RDP search tools.

\begin{tabular}{|c|c|c|c|c|c|}
\hline Type & Band no. & Closest relatives & Accession no. & Identity (\%) & Affiliation \\
\hline \multirow[t]{26}{*}{ Eubacteria } & 1 & Enterobacter sp. & KX914654.1 & 100 & Enterobacter \\
\hline & 2 & Enterobacter sp. & KY065507.1 & 100 & Enterobacter \\
\hline & 3 & Chryseobacterium sp. & KY117480.1 & 100 & Chryseobacterium \\
\hline & 4 & Uncultured Clostridium sp. & LC055608.1 & 100 & Clostridium \\
\hline & 5 & Staphylococcus sp. & KX881396.1 & 100 & Staphylococcus \\
\hline & 6 & Acetobacter pasteurianus & KX424632.1 & 98 & Acetobacter \\
\hline & 7 & Uncultured Rhodospirillaceae bacterium & LT625193.1 & 99 & bacterium \\
\hline & $8,21,27,28,29$ & Acetobacter pasteurianus & СР015168.1 & 99 & Acetobacter \\
\hline & 9 & Blautia sp. & LT631509.1 & 99 & Blautia \\
\hline & 10,12 & Uncultured bacterium & EU234163.2 & 100 & bacterium \\
\hline & 11 & Uncultured Lachnoanaerobaculum sp. & KU359740.1 & 100 & Lachnoanaerobaculum \\
\hline & 13 & Clostridium autoethanogenum & СР012395.1 & 100 & Clostridium \\
\hline & 14 & Uncultured Ruminococcus sp. & KX672523.1 & 99 & Ruminococcus \\
\hline & 15 & Uncultured Lactobacillus sp. & KU674948.1 & 100 & Lactobacillus \\
\hline & 16 & Uncultured bacterium & KJ853484.1 & 99 & bacterium \\
\hline & 17 & Clostridium kluyveri & LC149721.1 & 99 & Clostridium \\
\hline & 18 & Uncultured Clostridium sp. & JX575865.1 & 99 & Clostridium \\
\hline & 19 & Enterobacter ludwigii & KX959985.1 & 100 & Enterobacter \\
\hline & 20 & Acetobacter tropicalis & KX424645.1 & 99 & Acetobacter \\
\hline & 22 & Enterobacter asburiae & KU724380.1 & 100 & Enterobacter \\
\hline & 23 & Gamma proteobacterium & LC168577.1 & 100 & proteobacterium \\
\hline & 24,25 & Uncultured Caloramator sp. & KX672807.1 & 100 & Caloramator \\
\hline & 26 & Gluconobacter sp. & KX578017.1 & 100 & Gluconobacter \\
\hline & 30 & Uncultured Clostridium sp. & LC036237.1 & 100 & Clostridium \\
\hline & 31 & Uncultured Ruminococcus sp. & KX672726.1 & 99 & Ruminococcus \\
\hline & 32 & Acetobacter nitrogenifigens & KC763598.1 & 100 & Acetobacter \\
\hline
\end{tabular}

DGGE analysis on the quantitative results of microbes in the cellar. Overall, this study provides methods for quantitative analysis of the Clostridium genus and monitoring of their community dynamics in various fermentation systems.

\section{Acknowledgments}

We are grateful to the National Natural Science Foundation of China (31671833) and the Science and Technology Support Program of Sichuan Province (2015KJT022-2011SZ).

\section{Conflict of Interest}

The authors have no financial conflicts of interest to declare.

\section{References}

1. Wu Q, Chen L, Xu Y. 2013. Yeast community associated with the solid state fermentation of traditional Chinese Maotai-flavor liquor. Int. J. Food Microbiol. 166: 323-330.

2. Wang Q, Zhang H, Liu X. 2016. Microbial community composition associated with Maotai liquor fermentation. $J$. Food. Sci. 81: 1485-1494.

3. Wu YY, Guo SZ, Xue TR, Chen SR, Lu SY. 1993. Studying on the brewing microbes of Chinese traditional Luzhouflavor liquor. Liquor Making Sci. Technol. 5: 30-35.

4. Wu YY, Qi YP, Xue CJ, Guo SZ, Lu XJ. 1980. Microbial ecology distribution and anaerobic fermentation characteristics in the pit mud of Luzhou-flavor liquor. Microbiol. China 3: 108-112.

5. Hu X, Du H, Ren C, Xu Y. 2016. Illuminating anaerobic microbial community and cooccurrence patterns across a 
quality gradient in Chinese liquor fermentation pit muds. Appl. Environ. Microbiol. 82: 2506-2515.

6. Tao Y, Wang X, Li X, Wei N, Jin H, Xu Z, et al. 2017. The functional potential and active populations of the pit mud microbiome for the production of Chinese strong-flavour liquor. Microb. Biotechnol. 10: 1603-1615.

7. Zheng J, Liang R, Wu C, Zhou R, Liao X. 2014. Discrimination of different kinds of Luzhou-flavor raw liquors based on their volatile features. Food Res. Int. 56: 77-84.

8. Fan W, Qian MC. 2005. Headspace solid phase microextraction and gas chomatography-olfactometry dilution analysis of young and aged Chinese "Yanghe Daqu" liquors. J. Agric. Food Chem. 53: 7931-7938.

9. Fan W, Qian MC. 2006. Characterization of aroma compounds of Chinese "Wuliangye" and "Jiannanchun" liquors by aroma extract dilution analysis. J. Agric. Food Chem. 54: 2695-2704.

10. Fan W, Qian MC. 2006. Identification of aroma compounds in Chinese 'Yanghe Daqu' liquor by normal phase chromatography fractionation followed by gas chromatography/ olfactometry. Flavour Fragrance J. 21: 333-342.

11. Guth H. 1997. Quantitation and sensory studies of character impact odorants of different white wine varieties. J. Agric. Food Chem. 45: 3027-3032.

12. $\mathrm{Hu} \mathrm{XL}, \mathrm{Du} \mathrm{H}, \mathrm{Xu}$ Y. 2015. Identification and quantification of the caproic acid-producing bacterium Clostridium kluyveri in the fermentation of pit mud used for Chinese strong-aroma type liquor production. Int. J. Food Microbiol. 214: 116-122.

13. Tao $\mathrm{Y}, \mathrm{Hu} \mathrm{X}, \mathrm{Zhu} \mathrm{X}$, Jin $\mathrm{H}, \mathrm{Xu} \mathrm{Z}$, Tang Q, et al. 2016. Production of butyrate from lactate by a newly isolated Clostridium sp. BPY5. Appl. Biochem. Biotechnol. 179: 361-374.

14. $\mathrm{Hu} \mathrm{XL}$, Wang $\mathrm{HY}, \mathrm{Wu} \mathrm{Q}$, Xu Y. 2014. Development, validation and application of specific primers for analyzing the clostridial diversity in dark fermentation pit mud by PCR-DGGE. Bioresour. Technol. 163: 40-47.

15. Liu M, Zhao K, Tang Y, Ren D, Yao W, Tian X, et al. 2015. Analysis of Clostridium cluster I community diversity in pit mud used in manufacture of Chinese Luzhou-flavor liquor. Food. Sci. Biotechnol. 24: 995-1000.

16. Rudolf Amann, Bernhard M Fuchs, Behrens S. 2001. The identification of microorganisms by fluorescence in situ hybridisation. Curr. Opin. Biotechnol. 12: 231-236.

17. Moter A, Göbel UB. 2000. Fluorescence in situ hybridization (FISH) for direct visualization of microorganisms. J. Microbiol. Methods 41: 85-112.

18. Karakashev D, Batstone DJ, Angelidaki I. 2005. Influence of environmental conditions on methanogenic compositions in anaerobic biogas reactors. Appl. Environ. Microbiol. 71: 331-338.

19. Xia Y, Masse DI, McAllister TA, Kong Y, Seviour R, Beaulieu C. 2012. Identity and diversity of archaeal communities during anaerobic co-digestion of chicken feathers and other animal wastes. Bioresour. Technol. 110: 111-119.

20. Jupraputtasri W, Boonapatcharoen N, Cheevadhanarak S, Chaiprasert P, Tanticharoen M, Techkarnjanaruk S. 2005.
Use of an alternative Archaea-specific probe for methanogen detection. J. Microbiol. Methods 61: 95-104.

21. Crocetti G, Murto M, Bjornsson L. 2006. An update and optimisation of oligonucleotide probes targeting methanogenic Archaea for use in fluorescence in situ hybridisation (FISH). J. Microbiol. Methods 65: 194-201.

22. Li H, Huang J, Liu X, Zhou R, Ding X, Xiang Q, et al. 2016. Characterization of interphase microbial community in Luzhou-flavored liquor manufacturing pits of various ages by polyphasic detection methods. J. Microbiol. Biotechn. 27: 130-140.

23. Zhang L, Zhou R, Niu M, Zheng J, Wu C. 2015. Difference of microbial community stressed in artificial pit muds for Luzhou-flavour liquor brewing revealed by multiphase culture-independent technology. J. Appl. Microbiol. 119: 13451356.

24. Ding X, Wu C, Huang J, Li H, Zhou R. 2014. Eubacterial and archaeal community characteristics in the man-made pit mud revealed by combined PCR-DGGE and FISH analyses. Food Res. Int. 62: 1047-1053.

25. Wu C, Ding X, Huang J, Zhou R. 2015. Characterization of archaeal community in Luzhou-flavour pit mud. J. I. Brewing. 121: 597-602.

26. Weimer PJ, Stevenson DM. 2012. Isolation, characterization, and quantification of Clostridium kluyveri from the bovine rumen. Appl. Microbiol. Biotechnol. 94: 461-466.

27. Ding $X$, Wu C, Huang J, Zhou R. 2015. Interphase microbial community characteristics in the fermentation cellar of Chinese Luzhou-flavor liquor determined by PLFA and DGGE profiles. Food Res. Int. 72: 16-24.

28. Bertilsson S, Cavanaugh CM, Polz MF. 2002. Sequencingindependent method to generate oligonucleotide probes targeting a variable region in bacterial 16S rRNA by PCR with detachable primers. Appl. Environ. Microbiol. 68: 6077-6086.

29. Wang C, Esteve-Zarzoso B, Mas A. 2014. Monitoring of Saccharomyces cerevisiae, Hanseniaspora uvarum, and Starmerella bacillaris (synonym Candida zemplinina) populations during alcoholic fermentation by fluorescence in situ hybridization. Int. J. Food Microbiol. 191: 1-9.

30. Sekiguchi Y, Kamagata Y, Nakamura K, Ohashi A, Harada H. 1999. Fluorescence in situ hybridization using $16 \mathrm{~S}$ rRNAtargeted oligonucleotide reveals location of methanogens and selected uncultured bacteria in mesophilic and thermophilic sludge granules. Appl. Environ. Microbiol. 65: 1280-1288.

31. Amann RI, Binder BJ, Olson RJ, Chisholm SW, Devereux R, Stahl DA. 1990. Combination of 16S rRNA-targeted oligonucleotide probes with flow cytometry for analyzing mixed microbial populations. Appl. Environ. Microbiol. 56: 1919-1925.

32. Zhou J, Mary AB, Tiedje JM. 1996. DNA recovery from soils of diverse composition. Appl. Environ. Microbiol. 62: 316-322.

33. Lee C, Kim J, Shin SG, Hwang S. 2006. Absolute and relative QPCR quantification of plasmid copy number in Escherichia coli. J. Biotechnol. 123: 273-280. 
34. Whelan JA, Russell NB, Whelan MA. 2003. A method for the absolute quantification of cDNA using real-time PCR. J. Immunol. Methods 278: 261-269.

35. Quast C, Pruesse E, Yilmaz P, Gerken J, Schweer T, Yarza P, et al. 2013. The SILVA ribosomal RNA gene database project: improved data processing and web-based tools. Nucleic Acids Res. 41: D590-596.

36. Bouvier T, Del Giorgio PA. 2003. Factors influencing the detection of bacterial cells using fluorescence in situ hybridization (FISH): A quantitative review of published reports. FEMS Microbiol. Ecol. 44: 3-15.

37. Stahl D. 1991. Development and application of nucleic acid probes. Nucleic Acid Techniques in Bacterial Systematics. 8: 205-248.

38. Manz W, Amann R, Ludwig W, Wagner M, Schleifer K-H. 1992. Phylogenetic oligodeoxynucleotide probes for the major subclasses of proteobacteria: problems and solutions. Syst. Appl. Microbiol. 15: 593-600.

39. Snaidr J, Amann R, Huber I, Ludwig W, Schleifer K-H. 1997. Phylogenetic analysis and in situ identification of bacteria in activated sludge. Appl. Environ. Microbiol. 63: 2884-2896.

40. Knutsson R, Lofstrom C, Grage H, Hoorfar J, Radstrom P. 2002. Modeling of $5^{\prime}$ nuclease real-time responses for optimization of a high-throughput enrichment PCR procedure for salmonella enterica. J. Clin. Microbiol. 40: 52-60.

41. Klein D. 2002. Quantification using real-time PCR technology: applications and limitations. Trends Mol. Med. 8: 257-260.

42. Kim J, Lim J, Lee C. 2013. Quantitative real-time PCR approaches for microbial community studies in wastewater treatment systems: applications and considerations. Biotechnol. Adv. 31: 1358-1373.

43. Fittipaldi M, Codony F, Adrados B, Camper AK, Morato J. 2011. Viable real-time PCR in environmental samples: can all data be interpreted directly. Microb. Ecol. 61: 7-12.

44. Yu Y, Lee C, Kim J, Hwang S. 2005. Group-specific primer and probe sets to detect methanogenic communities using quantitative real-time polymerase chain reaction. Biotechnol. Bioeng. 89: 670-679.

45. Bornstein BT, Barker HA. 1948. The nutrition of Clostridium kluyveri. J. Bacteriol. 55: 223-230. 